\title{
Occurrence of Insulin Resistance with Recurrent Pregnancy Loss of Unknown Etiology in North Indian Hospital-based Women Population: A Pilot Study
}

\author{
Harpreet Singh ${ }^{1}$, Anubhuti Chitkara ${ }^{2}$, Manju Puri ${ }^{3}$, Binita Goswami ${ }^{4}$, Jayashree Bhattacharjee ${ }^{5}$
}

\begin{abstract}
Objective: Recurrent pregnancy loss (RPL) has been defined as occurrence of two or more clinically recognized spontaneous consecutive pregnancy losses prior to the 20th gestational weeks according to new guidelines of the American Society of Reproductive Medicine. Although multiple factors have been explained for RPL but still in most of the cases no etiology is found.

Study design: It was a prospective analytical observational study. It was conducted on 100 nonpregnant women in reproductive age-group 20-30 years, 50 women, with history of two or more consecutive spontaneous abortions in study group and 50 women with at least one live birth and no history of recurrent abortions in control group. These two groups were matched for age and BMI. All the women underwent various examinations like ultrasound pelvis for ruling out any uterine anomaly, hysteroscopy, antiphospholipid profile, hormonal profile, screening for DM, and thyroid disorders, HOMA-IR was calculated for insulin resistance.

Result: A significant difference $(<0.0001)$ was observed in fasting blood sugar and serum insulin levels in the case and control groups $(<0.0005)$. HOMA-IR was also found to be significantly increased in RPL group $(p \leq 0.0005)$.

Conclusion: Insulin resistance and hyperinsulinemia may be an important risk factor in women with idiopathic RPL.

Keywords: HOMA-IR, Insulin resistance, Pregnancy outcome, Recurrent pregnancy loss (RPL), Unexplained etiology.

International Journal of Infertility and Fetal Medicine (2022): 10.5005/jp-journals-10016-1256
\end{abstract}

\section{INTRODUCTION}

Many human conceptions end in miscarriage and recurrent pregnancy (RPL) loss is a common complication of pregnancy. RPL is defined as loss of two or more consecutive and clinically recognized pregnancies before 20 weeks' gestation. ${ }^{1}$ Most physicians recommend evaluation after two consecutive losses. ${ }^{2}$ The American Society of Reproductive Medicine (ASRM) defines recurrent pregnancy loss as two or more pregnancy losses and recommends early investigation into the etiology after two losses as it is likely to result in continuation of pregnancy with fruitful conception.

The incidence of recurrent pregnancy loss is $2-5 \%$ of all couples in the reproductive age-group. ${ }^{3}$ Epidemiological studies have suggested that the disease might be multifactorial, with a possible genetic predisposition and involvement of environmental factors in its pathogenesis. ${ }^{4}$ Anatomic abnormalities contribute to $10-15 \%$ cases of RPL. ${ }^{5}$ Luteal phase defect (LPD), polycystic ovarian syndrome (PCOS), diabetes mellitus, thyroid disease, and hyperprolactinemia are the most common endocrinological disorders implicated in approximately $17-20 \%$ of RPL cases. ${ }^{6}$ Approximately $2-4 \%$ of RPL is associated with a parental balanced structural chromosome rearrangement. ${ }^{5}$ Other known causes of RPL include antiphospholipid antibodies (APA), and thrombophilias. ${ }^{7}$ Unfortunately, standard evaluation for cause of RPL typically leave one-half of patients with this disorder unexplained. ${ }^{\text {? }}$

Evaluation of certain population groups that have a higher incidence of idiopathic pregnancy loss have suggested other potential etiologies like hyperinsulinemia, insulin resistance, and increased plasminogen activator inhibitor activity for
'Department of Obstetrics and Gynaecology, LHMC, Delhi, India

2Department of Biochemistry, Maulana Azad Medical College, New Delhi, India

${ }^{3}$ Department of Obstetrics and Gynaecology, Lady Hardinge Medical College and Smt Sucheta Kriplani Hospital, Delhi, India

${ }^{4}$ Department of Biochemistry, Lady Hardinge Medical College, Delhi, India

${ }^{5}$ Department of Biochemistry, Lady Hardinge Medical College and Smt Sucheta Kriplani Hospital, Delhi, India

Corresponding Author: Anubhuti Chitkara, Department of Biochemistry, Maulana Azad Medical College, New Delhi, India, Phone: +91 9999323592, e-mail: anubhuti1234@rediffmail.com

How to cite this article: Singh H, Chitkara A, Puri M, et al. Occurrence of Insulin Resistance with Recurrent Pregnancy Loss of Unknown Etiology in North Indian Hospital-based Women Population: A Pilot Study. Int J Infertil Fetal Med 2022;13(1):15-17.

Source of support: Nil

Conflict of interest: None

unexplained recurrent pregnancy loss. Insulin resistance is a condition in which cell receptors which are the target site for insulin action do not respond to the hormone insulin and resultant hyperinsulinemia. ${ }^{8}$ Insulin resistance is often associated with a hypercoagulable state (impaired fibrinolysis) and increased inflammatory cytokine levels. ${ }^{9}$

In view of this, we planned this study aiming to investigate the prevalence of IR and hyperinsulinemia in patients with RPL with unknown etiology. 


\section{Materials and Methods}

This was an observational, hospital-based, prospective analytical study conducted on 100 nonpregnant women in the reproductive age-group. The study was approved by the Institutional Ethics Committee and written informed consent was obtained from all the patients. These women were divided into two groups, Group A (Study group), comprising 50 nonpregnant women in the agegroup of 20-35 years with history of two or more consecutive spontaneous abortions of unexplained etiology. The exclusion criteria included ongoing pregnancy, obesity, Diabetes Mellitus, PCOS, Hypertension, and any other ongoing medical disorder. Group B (control group) fulfilled the same selection and exclusion criteria except that these women had at least one previous live baby, no history of recurrent spontaneous abortions, and no history suggestive of gestational D.M., for example, big-sized baby, hydramnios, etc.

The two groups were matched for age and BMI. All the women underwent following examinations, viz. ultrasonography, hysterosalpingography or hysteroscopy, serum thyroid profile, prolactin, LH/FSH ratio, APLA screening. The women with any known etiology were excluded. Sixteen women were excluded from the study group. The blood samples for fasting glucose and fasting insulin were collected after 12 hours fasting in plain tubes. Blood samples were centrifuged immediately after clot formation. The fasting glucose was determined soon after the separation of serum. Blood glucose was determined by GOD/POD method. For fasting insulin levels, serum was kept in aliquots at about $-20^{\circ} \mathrm{C}$ (in deep freeze) and it was determined by ELISA.

We used the homeostasis model assessment of insulin resistance (HOMA-IR) index in both groups. HOMA IR = FG $(\mathrm{mg} / \mathrm{dL})$ $x \mathrm{FI}(\mathrm{IU} / \mathrm{mL}) / 405$. Fasting insulin of $>7.5 \mathrm{IU} / \mathrm{mL}$ was considered as hyperinsulinemia and a cut-off value of 1.5 for HOMA-IR being considered as diagnostic for insulin resistance. ${ }^{10}$

The results were analyzed by SPSS version 22. Continuous variables were presented as mean \pm standard deviation. Value less than 0.05 was considered statistically significant.

\section{Observations and Results}

In this study, both the groups were matched for age and BMI. The mean age of women was $25.1 \pm 3.0$ years and $25.8 \pm 2.8$ years in case and control groups, respectively with no statistically significant difference $(p=0.249)$. The mean BMl in group A was $21.91 \pm 1.98$ and in group $B$ was $21.47 \pm 2.52$ which is also statistically insignificant.

Fasting blood glucose was estimated in both the groups. The women in both groups were in euglycemic range with mean fasting blood sugar $83.82 \pm 6.51 \mathrm{mg} / \mathrm{dl}$ in study group and $75.26 \pm 7.43 \mathrm{mg} / \mathrm{dl}$ in control group, $p$ value was statistically significant $(<0.0001)$.

The mean fasting insulin was $10.13 \pm 6.47 \mu \mathrm{lU} / \mathrm{mL}$ and $5.43 \pm$ $3.45 \mu \mathrm{lU} / \mathrm{mL}$ in group $\mathrm{A}$ and group $\mathrm{B}$, respectively. The difference was found to be statistically significant.

Mean HOMA-IR was $2.07 \pm 1.27$ in the study group and $1.02 \pm$ 1.07 in the control group the difference was statistically significant (Tables 1 and 2).

\section{Discussion}

In this study it was observed that both insulin resistance and hyperinsulinemia were significantly associated with recurrent
Table 1: Demographic profile of study and control groups

\begin{tabular}{lccc}
\hline Parameters & $\begin{array}{c}\text { Study group } \\
\text { (Group A) } \\
\text { Mean } \pm \text { SD }\end{array}$ & $\begin{array}{c}\text { Control group } \\
\text { (Group B) } \\
\text { Mean } \pm \text { SD }\end{array}$ & p-value \\
\hline Age (years) & $25.1 \pm 3.0$ years & $25.8 \pm 2.8$ years & 0.249 \\
Pregnancy loss & $2.74 \pm 0.98$ & $0.1 \pm 0.2$ & $<0.0001$ \\
BMI & $21.91 \pm 1.98$ & $21.47 \pm 2.52$ & 0.334 \\
Fasting glucose & $83.82 \pm 7.51$ & $75.26 \pm 6.43$ & $<0.0001$ \\
(mg/dl) & & & \\
$\begin{array}{l}\text { Fasting insulin } \\
\text { (IU/mL) }\end{array}$ & $10.13 \pm 6.47$ & $5.73 \pm 3.45$ & $<0.0005$ \\
HOMA-IR & $2.07 \pm 1.27$ & $1.02 \pm 1.07$ & $<0.0005$ \\
\hline
\end{tabular}

Table 2: Comparison of insulin resistance in two groups based on fasting insulin levels and HOMA IR

\begin{tabular}{lcccc}
\hline \multirow{2}{*}{ Fasting insulin $(\mathrm{IU} / \mathrm{mL})$} & \multicolumn{3}{c}{ Cases (34) } & Control (50) \\
\cline { 2 - 5 } & No & $\%$ & No. & $\%$ \\
\hline$>7.5$ & 21 & $61.8 \%$ & 6 & $12 \%$ \\
$<7.5$ & 13 & $38.2 \%$ & 44 & $88 \%$ \\
HOMA-IR & & & & \\
$>1.5$ & 22 & $64.7 \%$ & 6 & $12 \%$ \\
$<1.5$ & 12 & $35.3 \%$ & 44 & $88 \%$ \\
\hline
\end{tabular}

pregnancy loss. It appears to be similar to the insulin status of the women with polycystic ovarian syndrome.

Hyperinsulinemia adversely affects the preimplantation environment by decreasing the expression of glycodelin and IGF-binding protein $-1^{11}$ which may play a role in inhibiting the endometrial immune response of the embryo, and seems to facilitate adhesion processes at the feto-maternal interface.

In our study, there was a statistically significant difference in the fasting glucose levels $(83.82 \pm 7.51 \mathrm{mg} / \mathrm{dL}, 75.26 \pm$ $6.43 \mathrm{mg} / \mathrm{dL})$ and fasting serum insulin levels $(10.13 \pm 6.4 \mu \mathrm{IU} / \mathrm{mL}$, $5.73 \pm 3.45 \mu \mathrm{lU} / \mathrm{mL}$ ) in case vs control groups. Similar findings have been reported by Wani et al., ${ }^{12}$ Celik Nazan et al., ${ }^{13} \mathrm{KA}$ Wahba et al., ${ }^{14}$ and Wang $Y$ Zhao et al. ${ }^{15}$ The HOMA- IR value in our study was $2.07 \pm 1.27$ in the cases group and $1.02 \pm 1.07$ in control group which is in congruence with the findings of the study done by Corrina-Alina et al. ${ }^{16}$ where mean HOMA- IR in the study group was 2.98 and in the control group was 1.69 with a significant $p$ value $<0.001$

\section{Conclusion}

It is concluded that Insulin resistance is associated with RPL with unexplained etiology when fasting blood glucose, fasting insulin and HOMA-IR are used to measure insulin resistance. More studies are needed to explore the mechanism of insulin resistance in pregnancy loss.

\section{References}

1. Practice Committee of American Society for Reproductive Medicine. Definitions of infertility and recurrent pregnancy loss: a committee opinion. Fertil Steril 2013;99(1):63. DOI: 10.1016/j. fertnstert.2012.09.023

2. Grattan D, Ladyman S. Hormonal induction of leptin resistance during pregnancy. Physiol Behav 2007;91(4):366-374. DOI: 10.1016/j.physbeh.2007.04.005 
3. Practice Committee of the American Society for Reproductive Medicine. Evaluation and treatment of recurrent pregnancy loss: a committee opinion. Fertil Steril 2012;98(5):1103-1111. DOI: 10.1016/j. fertnstert.2012.06.048

4. Jeddi-Tehrani M, Torabi R, Mohammadzadeh A, et al. Investigating association of three polymorphisms of coagulation factor XIII and recurrent pregnancy loss. Am J Reprod Immunol 2010;64(3):212-217. DOI: 10.1111/j.1600-0897.2010.00838.x

5. Ford BH, Schust DJ. Recurrent pregnancy loss: etiology, diagnosis, and therapy. Rev Obstet Gynecol 2009;2(2):76-88.

6. Mtiraoui N, Borgi L, Hizem S, et al. Prevalence of antiphospholipid antibodies, factor V G1691A (Leiden) and prothrombin G20210A mutations in early and late recurrent pregnancy loss Eur JObstet Gynecol Reprod Biol 2005;119(2):164-170. DOI:10.1016/j.ejogrb.2004.07.00

7. Stephenson MD. Frequency of factors associated with habitual abortion in 197 couples. Fertil Steril 1996;66(1):24-29.

8. Jakubowicz DJ, Essah PA. Reduced serum glycodelin and insulin like growth factor binding protein-1 in women with polycystic ovary syndrome during first trimester of pregnancy. J Clin Endocrinol Metab 2004;89(2):833-839. DOI: 10.1210/jc.2003-030975

9. Craig LB, Ke RW, Kutteh WH. Increased prevalence of insulin resistance in women with a history of recurrent pregnancy loss Fertil Steril 2002;78(3):487-490. DOI: 10.1016/s0015-0282(02)03247-8
10. Kulshreshtha B, Ganie MA, Praveen EP, et al. Insulin response to oral glucose in healthy, lean young women and patients with polycystic ovary syndrome. Gynecol Endocrinol 2008;24(11):637-643. DOI: 10.1080/09513590802342858

11. Giudice LC. Endometrium in PCOS: Implantation and predisposition to endocrine CA. Best Pract Res Clin Endocrinol Metab 2006;20(2): 235-244. DOI: 10.1016/j.beem.2006.03.005

12. Wani AA, Gul I, Jabeen F, et al. Relationship of insulin resistance with recurrent pregnancy loss. Int J Reprod Contracept Obstet Gynecol 2017;6(4):1312-1317. DOI: 10.18203/2320-1770. ijrcog20171384

13. Nazan C, Evsen MS, Sak ME, et al. Evaluation of the relationship between insulin resistance and recurrent pregnancy loss. Ginekol Pol 2011;82(4):272-275.

14. Wahba KA. Assessment of the role of insulin resistance in cases of recurrent unexplained first trimesteric miscarriage. Life Sci J 2015;12(7):174-181.

15. Wang Y, Zhao H, LiY, et al. Relationship between recurrent miscarriage and insulin resistance. Gynecol Obstet Invest 2011;72(4):245-251. DOI: 10.1159/000325165

16. Ispasoiu CA, Chicea R, Stamatian FV, et al. High fasting insulin levels and insulin resistance may be linked to idiopathic RPL. Int J Endocrinol 2013;2013:576926. DOI: 10.1155/2013/576926 